\title{
Supporting information to: Single layer deposition of polystyrene particles onto planar Polydimethylsiloxane substrates
}

\section{Fabrication of flow cell and substrate preparation}

The flow cell was fabricated by using standard PDMS soft lithography. Micromold masters for the flow cell were produced in a cleanroom using standard SU-8 (MicroChem, Newton, MA, US) photolithography with typical feature heights of $100 \mu \mathrm{m}$. A negative of the mask that was used for the process is shown in Figure 1a. In order to achieve the desired flow chamber dimensions (see Figure 1b) and inlet hole, small disk shaped parts with a pin in the center were machined from aluminum and bonded onto the micromold.

In the next fabrication step, Sylgard 184 PDMS base and hardener were combined at a base : hardener ratio of 10:1 and subsequently mixed in two steps: First manual mixing was performed for 3 minutes by using a clean metal spatula. Subsequently, the pre-mixed PDMS was put in a AR250 (Thinky) vortex mixer (3 minutes mixing and 1:30 minutes degassing).

The mixture was poured over the mold master and then put in a desiccator for further degassing. Subsequently, the PDMS covered mold was put in an oven to cure at $65{ }^{\circ} \mathrm{C}$ for 5 hrs. Finally, the cured PDMS was peeled from the mold, cut into shape and the fluid inlet and outlet holes were punched.

Substrates were prepared by pouring Sylgard 184 in 10:1 (base : hardener, same mixing procedure) over a microscope slide and subsequent degassing. The work was performed under a laminar flow hood to minimize dust deposition on the substrate. The substrates were then cured at $65^{\circ} \mathrm{C}$ for $5 \mathrm{hrs}$. The thickness of the PDMS layer over the glass slide varied between $0.5-1 \mathrm{~mm}$. At such film thickness, the cross-linking of PDMS is unaffected by the presence of the microscope slide [1]. Subsequently, the substrates were put in sealed petri dishes and stored in a dark cabinet until they were used for a deposition experiment. None of the substrates used for the experiments in this work was older than 3 days. We did not observe any correlation between substrate age and deposition rates. Finally, it is worth mentioning that cross-linked PDMS is nanoporous and water as well as small molecules can be absorbed by PDMS [2], [3], [4].

The impinging jet cell was completed by placing the flow cell on the substrate as shown in Figure 1c and subsequent mechanical clamping of these two parts as shown in Figure 1d. By 
assuming uniform compression of the PDMS device and by treating the clamp-plates as perfectly rigid, the typical resulting compression of the deposition chamber height $h$ due to clamping was estimated to be close to $1 \%$.

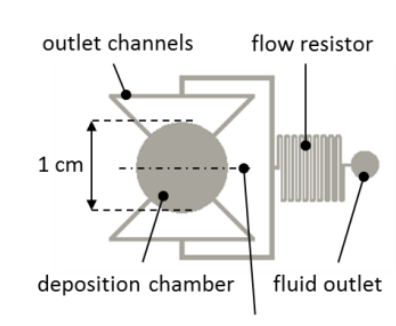

a)

$$
\text { cut-plane }
$$

b)

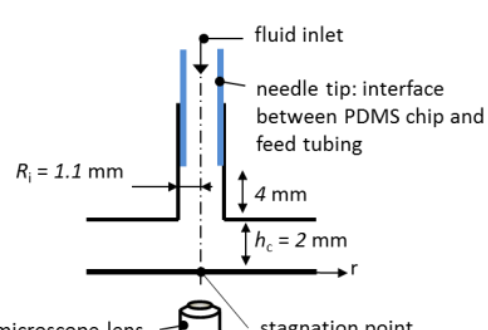

${ }^{m}$

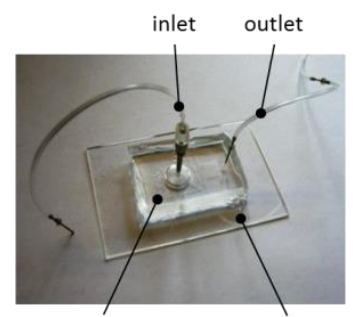

c)

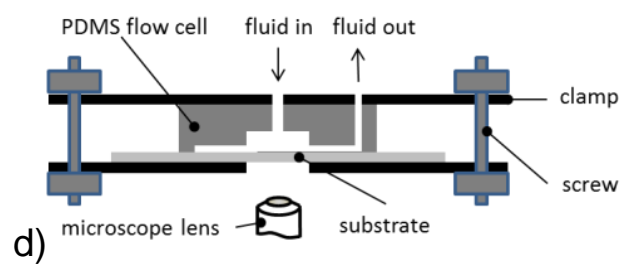

Figure 1: a) negative of the photomask used to fabricate mold masters for the flow cells; b) cross section view of the deposition chamber (cut plane is indicated in a)); c) flow cell on substrate without clamping; d) schematic of the clamped flow cell.

\section{Preparation of fluids}

An aqueous suspension of monodisperse, fluorescent polystyrene particles with a nominal mean diameter of $1 \mu \mathrm{m}$ and a density of $1053 \mathrm{~kg} / \mathrm{m}^{3}$ were purchased (Thermo Scientific, Waltham, MA, US). This suspension was dispersed in deionized water at $1 \%$ solid content and, according to the manufacturer, contained a small concentration in the order of $0.01 \mathrm{wt} \%$ of a surfactant consisting of a 12 carbon chain with a sulfate group at one end. Small amounts of the suspension provided by the manufacturer were first diluted with distilled water and then washed before they were further diluted by water and saline solutions to obtain the desired chemical conditions for the experiment.

Before washing, a small amount of the suspension provided by the manufacturer was diluted with Milli-Q ultrapure water to obtain a solid concentration of 0.01 wt\%. Subsequently, the diluted suspension was washed by using an accuspin 1R centrifuge (Fisher Scientific). Several washing steps were performed and after each step, the electrophoretic mobility of the suspension was measured by using a Zetasizer nano ZS 90. After three washing steps, the mobility decreased from $-6.3 \mu \mathrm{m} \cdot \mathrm{cm} /(\mathrm{V} \cdot \mathrm{s})$ to $-3.5 \mu \mathrm{m} \cdot \mathrm{cm} /(\mathrm{V} \cdot \mathrm{s})$ and subsequent washing steps 
did not result in a further change of the mobility. Thus it was concluded that all surfactant up to an insignificant amount had been removed by the three step washing procedure.

Some of the particles were accidentally removed from the suspension during the washing process. Before the washed particle suspension could be used to prepare the suspensions used for the experiments, the particle concentration had to be determined. Therefore small amounts of Tween 20 surfactant was added to samples of the washed suspension, which were then injected into a straight microchannel with known volume. The amount of surfactant added was kept small enough so that it had a negligible influence on the particle concentration to be measured. However, the surfactant did prevent particles from depositing in the concentration measurement channel. The microchannel used for concentration measurement was $400 \mu \mathrm{m}$ wide and $11 \mu \mathrm{m}$ deep. For each sample, 60 particle images were acquired as the suspension was flowing through the channel by using the Eclipse Ti microscope (Nikon) and Imager ProX2M camera (LaVision). The time between two image captures was large enough for all particles in the previous image to exit the field of view before the next image was taken. Subsequently, particles in each image were counted and the particle concentration was calculated using a custom Matlab script based on digital image processing methods that are described in section 6 of the supporting information. In order to minimize Poisson noise, the particle counts determined from all 60 images per sample were averaged. Furthermore, it was ensured that the depth of field exceeded the channel height so that all particles in the channel appeared approximately in focus on the image. With increasing particle concentration and channel height, the probability for the view on a particle to be blocked by another particle increases. However, it was ensured through numerical experiments that the concentration of particles and the channel height were low enough so that this effect could be neglected.

By using the procedure described above, 2 liters of washed particle suspension with a solid concentration of $0.0065 \mathrm{wt} \%$ was prepared. The washed particle suspension was stored in a Nalgene bottle in a refrigerator at $5^{\circ} \mathrm{C}$. It was then used to prepare all of the suspensions used in this work. All experiments were conducted during the course of two months. During this time, the stability of the washed suspension was monitored by performing visual inspections with our microscope setup (described further below). Particle agglomeration was not observed during the visual inspections. However, visual inspection of the Nalgene container walls (using a microscope) showed that particles deposited to the walls of the Nalgene container and, as a result, the concentration of dispersed particles in the container slightly decreased with increasing storage time. Hence, the concentration of the washed particle suspension was measured each time before it was used to prepare the suspensions for the deposition 
experiments. Before each particle deposition experiment, the suspensions used for the experiment were prepared by diluting the washed suspension further with biological certified sodium chloride (Fisher Scientific) saline solutions. The final solid concentration used for all

experiments in this work was $0.001 \mathrm{wt} \% \quad\left(n_{\mathrm{b}}=1.91 \cdot 10^{7} \frac{1}{\mathrm{~cm}^{3}}\right)$. The $\mathrm{pH}$ of all particle suspensions used in this work was determined to be between 6.3 and 6.6 by using an In-labflex micro $\mathrm{pH}$ electrode (Mettler Toledo).

\section{Particle and substrate zeta potentials}

The electrophoretic mobility of the particle suspensions used in this work was measured by using a Zetasizer Nano ZS-90 (Malvern) and DTS-1061 cuvettes. The Zetasizer measures the electrophoretic mobility of particles based on the electrophoretic light scattering technique. For high conductivity samples, the Zetasizer measures the mean electrophoretic mobility of the ensemble of particles in a given test volume. For low conductivity samples (here for $C_{\mathrm{NaCl}} \leq$ $10^{-2} M$ ), the Zetasizer provides the distribution of electrophoretic mobilities in the test volume, which is characterized by the corresponding standard deviation.

The mobility was measured for different salt concentrations. For each salt concentration, three suspensions were prepared (with a solid concentration of $0.001 \mathrm{wt} \%$ ) and subsequently one sample of each suspension was characterized three times. The $\mathrm{pH}$ of all samples was measured before starting the characterization by using an In-lab-flex micro $\mathrm{pH}$ electrode (Mettler Toledo). The $\mathrm{pH}$ of all samples varied between 6.3 and 6.6. The repeatability of the mean mobility measurement was excellent $(<5 \%$ deviation from mean mobility across all measurements) for all salt concentrations except for $C_{\mathrm{NaCl}}=0.5 \mathrm{M}$. At this salt concentration, the mobility was very low and it was difficult to measure low mobilities accurately.

The particle zeta potentials were derived from the measured electrophoretic mobilities by solving Ohshima's expression for the electrophoretic mobility [5]. The particle zeta potentials are shown as solid circles in Figure 2, where the error bars represent the mean of the standard deviation of the zeta potential across each sample.

The zeta potential $\xi_{\mathrm{i}}$ of the PDMS substrates was obtained by using the correlation equation

$$
\xi_{\mathrm{i}}=a_{0}+a_{1} \log _{10} C_{\mathrm{NaCl}}
$$

proposed by [6], where $C_{\mathrm{NaCl}}$ is the electrolyte concentration (in Molar), $a_{0}=6.75 \mathrm{mV}$ and $a_{1}=-29.75 \mathrm{mV}$ (for PDMS). The correlation equation was derived from electro osmosis and 
streaming potential experiments on Sylgard 184 PDMS (Dow Corning 10/1 (base/hardener)) that were performed at $6.5<\mathrm{pH}<7$ and for (1:1) electrolyte concentrations ranging from $10^{-4} \mathrm{M}$ to $10^{-2} \mathrm{M}$ and it is shown as dashed line in Figure 2. The present work, however, determines the PDMS zeta potential from eq (1) for salt concentrations ranging between $10^{-4} \mathrm{M}<C_{\mathrm{NaCl}}<0.5 \mathrm{M}$ at $6.3<\mathrm{pH}<6.6$ and the applicability of eq (1) for $C_{\mathrm{NaCl}}>10^{-2} \mathrm{M}$ is briefly discussed below.

A trend as described by eq (1) is in agreement with the Gouy-Chapman-Stern model for the electrical double layer, given that specific adsorption of counter ions on the substrate can be neglected and the zeta potential is large [7]. For example, the zeta potential of silica is well described by eq (1) for (1:1) electrolyte concentrations below $100 \mathrm{mM}$ at $\mathrm{pH}>6$ [7]. At larger salt concentrations (lower zeta potentials) eq (1) becomes invalid. More specifically, the zeta potential should approach zero with increasing electrolyte concentration instead of changing its sign. Consequently, the absolute values for the zeta potential of PDMS for $C_{\mathrm{NaCl}}>10^{-2} \mathrm{M}$ used in this work are inaccurate and most likely smaller than the corresponding true absolute zeta potentials. In other words, the height of the energy barriers in the interaction potentials for $C_{\mathrm{NaCl}}>10^{-2} \mathrm{M}$ is in fact larger than was assumed for the prediction of the concentration profiles and deposition rates. Further discussion on the validity of eq (1) can be found in [7].

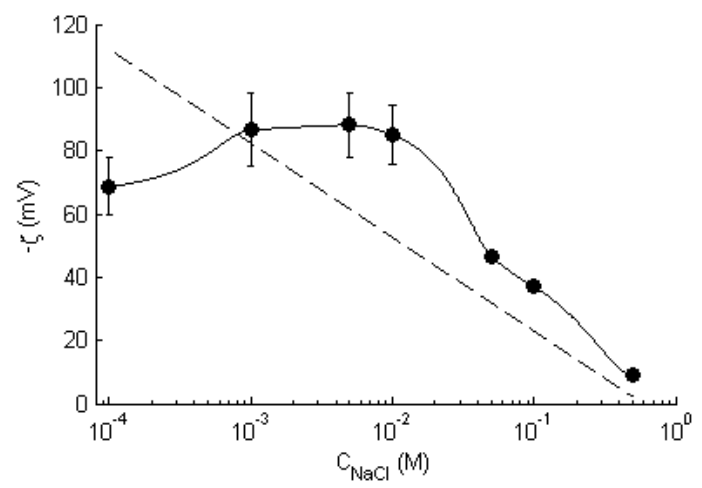

Figure 2: Zeta Potentials over salt concentration: Polystyrene particles (circles) and interpolant (solid line). The error bars (where available) represent the standard deviation of the particle zeta potential distribution across the particle population; PDMS zeta potential (dashed line) according to literature [6]. The $\mathrm{pH}$ of the all particle suspensions used in the current work was between 6.3 and 6.6.

\section{$4 \quad$ Experimental setup and procedure}

The experimental procedure used in this work can be subdivided in 3 steps 


\subsection{Assembling, filling and calibration of the impinging jet cell}

The flow cell was formed by clamping the PDMS device to the substrate as shown in Figure 1d. The impinging jet cell was filled with ultra-pure water; then the cell was connected to Tygon tubing that has also been filled with the same ultra-pure water. AFD steel needle tips served as interface between the impinging jet cell and tubing as indicated by the filled circles in Figure 3a. The needle tips were also used to connect the syringe pump to the setup. Before each experiment, the flow resistance of the impinging jet cell was determined by using the setup shown in Figure 3a. A syringe-pump (KD Scientific) drove fluid at a flow rate of $0.15 \mathrm{ml} / \mathrm{min}$ through the device while the pressure drop was measured by a ASDXRRX015PDAA5 differential pressure sensor (Honeywell). A typical pressure drop across the device at this flow rate was $360 \mathrm{mbar}+/-20 \%$.

\subsection{Particle deposition experiment}

Once the desired pressure drop over the device was determined, the flow was stopped and the syringe pump was exchanged for a pressure source and a reservoir containing the particle suspension as shown in Figure 3b. Instead of driving the flow with a syringe pump which allows specifying flow rate in a direct manner, the flow was driven by a pressure source in order to avoid unwanted pressure fluctuations caused by the stepper motor of the syringe pump [8]. The flow cell was placed on the stage of an inverted epi-fluorescence microscope equipped with CCD camera. The flow-rate was set to $Q=0.15 \mathrm{ml} / \mathrm{min}$ (corresponds to $R e=0.75$ and $P e=$ $1.3 \cdot 10^{-4}$ ) by adjusting the applied pressure until the desired pressure drop over the device was achieved. The CCD camera was then set to display images of the particles as they were transported towards the substrate by the flow and the field of view $(0.658 \mathrm{~mm} \times 0.877 \mathrm{~mm})$ was centered on the stagnation point, which is easily identified from the displayed image sequence. The first particle that deposited on the substrate was used as guide for focusing on the substrate, which was otherwise difficult to identify in the images. As shown in section 8 (supporting information), 15 minutes after initialization of the flow, steady state concentration profiles were expected and image acquisition was initialized and a series of images was recorded at a constant frame rate of $0.1 \mathrm{fps}$ while particles deposited on the substrate. After the experiment, these images were evaluated by a custom Matlab script that determines the initial particle deposition rate from the image sequence. The image processing procedure used in this work is described in section 6 . After $15-30$ minutes, the flow and the acquisition was stopped, then the whole system was flushed with particle free, ultra-pure water. 


\subsection{Removal of particles from substrate}

In the current work, several deposition experiments were performed on the same substrate. Before a substrate could be re-used, deposited particles had to be removed from the substrate. In order to do so, the impinging jet cell (filled with particle-free fluid) was disconnected from the tubing and carefully removed from the clamp without displacing the PDMS flow cell relative to the substrate.

Particles were removed from the substrate by passing a liquid-air interface over the substrate [9], [10], [11]. This was achieved by pressing one end of the flow cell to the substrate and lifting the other end by using tweezers as shown in Figure 3c. Subsequently, the substrate was briefly (< 10 seconds) dipped into a beaker that was immersed in a sonicator bath (Figure $3 \mathrm{~d}$ ). The beaker contained the same ultra-pure water that was used to prepare all other fluids in this work and the water was replaced after each use. The particle removal procedure described here removed the majority of deposited particles from the substrate without altering the position of the flow cell relative to the substrate. The small number of particles $(<100)$ that remained on the substrate after the cleaning procedure were expected to have negligible influence on the next deposition experiment. 


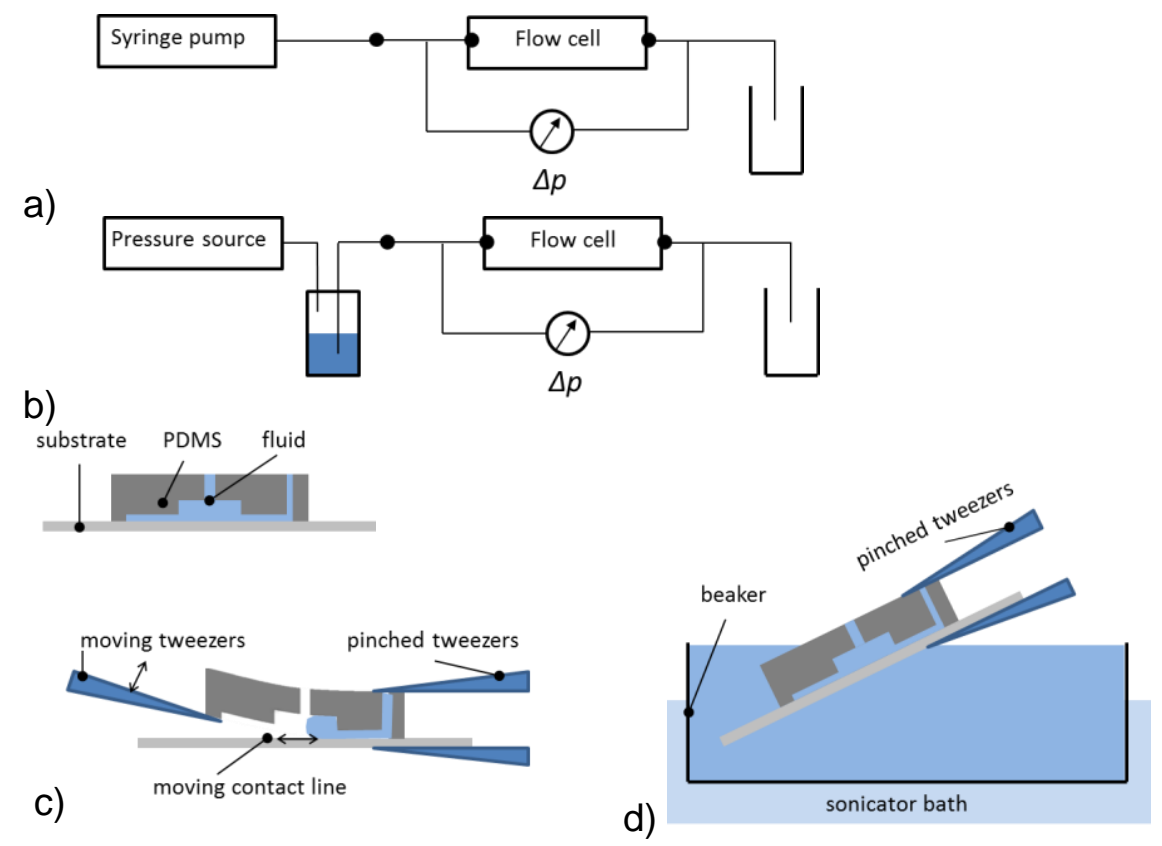

Figure 3: Schematic of the experimental setup a) for the calibration step and b) for the particle deposition experiment. The thin lines represent Tygon tubing and the black dots to steel needle tips that serve as tubing-tubing interface as well as flow-cell-tubing interface; c) schematic of particle removal from the substrate by passing a moving contact line over the substrate; d) schematic of particle removal from the substrate by using a sonicator.

\section{$5 \quad$ Surface roughness of the PDMS substrates}

The surface roughness of our PDMS substrates has been characterized from surface profiles of a representative sample that has been fabricated by using the method described in section 1 of the supporting information. Large scale surface profiles were obtained by using a NT-1100 (Veeco) optical profilometer (phase shifting interferometry) while surface profiles on a smaller scale were determined by using an MFP3D-SA (Asylum Research) Atomic Force Microscope (AFM) with a AC240TS (Asylum Research) silicon tip in tapping mode.

In order to reduce noise in all surface profiles, 3-5 surface profiles of a given region on the substrates were averaged and the averaged profiles were subsequently filtered by using a Gaussian Filter kernel with a standard deviation of 1 pixel. The resulting averaged profiles are shown in Figure 4. 
a)

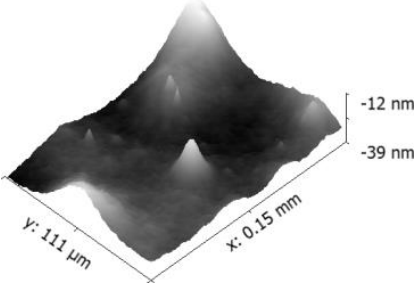

b)

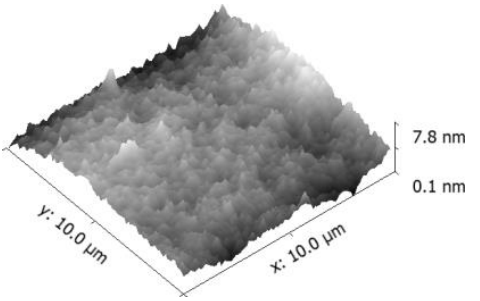

c)

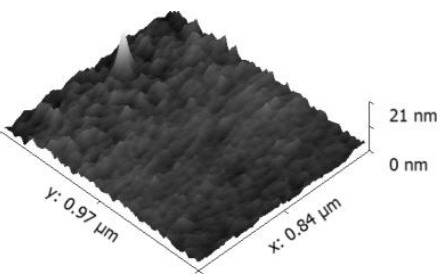

Figure 4: Surface profiles of a PDMS substrate surface: a) average of 5 large scale profiles obtained from optical profilometer, b) average of 3 intermediate scale profiles obtained from AFM and c) average of 4 small scale profiles obtained from AFM.

The root mean square surface roughness

$$
R_{\mathrm{q}}=\sqrt{\frac{1}{N} \sum_{w=1}^{N} z_{w}^{2}}
$$

and the root mean square wavelength $\lambda_{\mathrm{q}}$

$$
\lambda_{\mathrm{q}}=\frac{2 \pi \sqrt{\sum_{w=1}^{N} z_{w}^{2}}}{\sqrt{\frac{1}{\Delta x^{2}} \sum_{w-1}^{N}\left(z_{w+1}-z_{w}\right)^{2}}}
$$

of the profile were determined from the surface profiles by using the open source software Gwyddion. In equations (2)-(3), $N$ is the number of data points in the dataset, $z_{w}$ is the vertical height of datapoint (index $w$ ) measured from the mean height of the profile and $\Delta x$ is the spacing between two adjacent data points. Where values for $R_{\mathrm{q}}$ were determined from the entire 2 dimensional profiles, the wavelength $\lambda_{\mathrm{q}}$ was determined from a 1 dimensional subprofile along the diagonal from the bottom to the top of the profiles shown in Figure 4 . The resulting roughness parameters are listed in Table 1. 
Table 1: Representative surface roughness parameters for the PDMS substrates used in this work

\begin{tabular}{|l|l|l|l|}
\hline Field of view & $R_{\mathrm{q}}(\mathrm{nm})$ & $\lambda_{\mathrm{q}}(\mu \mathrm{m})$ & Pixel size $(\mathrm{nm})$ \\
\hline $\begin{array}{l}111.4 \mu \mathrm{m} \times \\
148.5 \mu \mathrm{m}\end{array}$ & 4.36 & 84.8 & 232 \\
\hline $10 \mu \mathrm{m} \times 10 \mu \mathrm{m}$ & 1.08 & 1.04 & 40 \\
\hline $\begin{array}{l}965 \mathrm{~nm} \times 835 \\
\mathrm{~nm}\end{array}$ & 1.13 & 0.14 & 4 \\
\hline
\end{tabular}

\section{$6 \quad$ Image acquisition and particle tracking}

All images were acquired under identical conditions. The optical system used in this work consisted of an Eclipse $\mathrm{Ti}$ inverted epi-fluorescence microscope (Nikon) equipped with a 20x Plan Fluor NA = 0.5 objective lens (Nikon), an imager ProX2M (LaVision) CCD camera and a 41002-TRITC filter cube (Chroma Technology Corp). The excitation light was provided by an Intensilight (Nikon) light source that was set to the lowest intensity possible (ND =1) in order to minimize photobleaching during the course of an experiment. The framerate was 0.1 frames per second (fps) and the exposure time was $600 \mathrm{~ms}$ for all experiments. In the obtained 14 bit gray-scale images with a resolution of $1600 \times 1200$ pixel, particles appear as bright and the background as dark. The pixel size was $0.548 \mu \mathrm{m} /$ pixel and the stagnation point was in the center of the images so that the area covered by the field of view was within $r / R_{\mathrm{i}}<0.5$ where the approximations equations (2)-(3) of the main article are valid. The intensity distribution of individual particles obtained from the optical system used in this work is well approximated by a Gaussian [12].

The image processing and particle tracking procedure used in this work follows classical methodology [13]. All images were smoothed by using a $3 \times 3$ pixel Gaussian blur filter (standard deviation 0.5 pixel) and all intensity values below a threshold (here 550 for the 14-bit grey scale resolution) were set to zero by using Matlab (Matworks). This particular intensity threshold value was chosen to remove the dark current and particles that are strongly out of focus from the images. Next, the positions of particles corresponding to local intensity maxima were detected by using the Matlab function imregionalmax. Subsequently, subpixel accuracy was achieved by finding the brightness weighted centroid for each particle [13]. The current 
work utilizes the particle tracking method by [14]. In brief, a proximity matrix containing Gaussian weighted distances for all possible links between located particles of an image pair is constructed. The singular value decomposition (SVD) of the proximity matrix then contains the information of a set of $i$ particle links with length $\delta_{i}$, which effectively represents a minimum length mapping [14]. The only free parameter in this tracking algorithm is the standard deviation (= 24 pixel) used for creating the proximity matrix.

In order to maximize the success rate for tracking a deposited (immobile) particle, a simple proximity search is applied before the SVD-based algorithm is used: For each particle at image position $\vec{X}_{i}$ in image one, we assign the closest neighbor in image two in a $3 \times 3$ pixel neighborhood around $\vec{X}_{i}$ as a match. As it will be shown further below, this pre-tracking step does not introduce a significant number of bad matches at the low image densities of moving particles given in this work. Each particle tracking step is finalized by simple outlier detection where all displacements larger than 90 pixel are discarded as invalid matches.

The performance of our image processing and tracking procedure was tested by using computer generated particle image pairs that closely resemble a pair of sequential experimental images.

Images of individual particles at any distance from the object plane are quantitatively captured by a Gaussian image intensity distribution as described in [12]. An image of many particles is then obtained based on the simplifying assumption that images of individual particles at different locations in the physical domain are additive. Furthermore, for each pixel with intensity I a random intensity value was first sampled from a normal distribution with standard deviation $\sigma_{N}(I)$ and subsequently added to the intensity value of that pixel to represent camera noise. The noise characteristic $\sigma_{N}\left(I_{\mathrm{av}}\right)$ of the camera had been determined experimentally from uniform grey images with average intensity $I_{\mathrm{av}}$. For a given set of particle coordinates in the physical domain, the model outlined here produced synthetic particle images that match the corresponding experimental particle images well in terms of particle image size, particle image intensity, depth of view, pixel resolution and noise. A qualitative comparison of a particle image obtained from our optical setup and a corresponding synthetic particle image is shown in Figure 5.

Sets of particle coordinates in a virtual physical domain above the substrate were produced by a simple dynamic simulation. First, a realistic number $n_{\mathrm{b}} V_{V}$ of mobile particles was distributed inside the virtual physical domain with Volume $V_{V}$ starting $1 \mu \mathrm{m}$ above the substrate. In addition to the mobile particles, a certain number of virtual deposited particles are distributed across the 
substrate. All particle positions were generated using the Matlab function rand to achieve the desired concentration of randomly distributed particles. Next, the positions of the mobile particles were advanced over a total time period of $\Delta \mathrm{t}=10$ seconds (with $\Delta t_{\text {step }}=0.1 \mathrm{~s}$ time steps) by using a simple explicit Euler forward time stepping scheme. At each time step, each coordinate of the position of a particle was advanced by a portion resulting from convection based on equations (2)-(3) of the main article and a portion due to Brownian motion that was sampled from a normal distribution with standard deviation $\sqrt{2 D_{0} \Delta t_{\text {step }}}$. Particles penetrating the virtual substrate were forced back to the substrate after each time step. Initial and final positions of the particles were stored in an array of reference trajectories. A synthetic particle image pair was then generated from the initial and final coordinates of all particles in the virtual domain. Subsequently, the tracking algorithm described above was applied to find particle trajectories in the synthetic image pairs. Particle tracking is most challenging at high particle image densities. Hence, the performance of the algorithm was tested for the worst case scenario where 2000 deposited particles have already deposited on the substrate. The number of deposited particles did not exceed 2000 in any of the laboratory experiments performed in this work.

The tracking efficiency for mobile particles $\beta_{m}\left(z_{\mathrm{e}}\right)$ is defined as the number of successful matches between tracking results and reference trajectories of mobile particles in $z<z_{\mathrm{e}}$ divided by the number of reference trajectories of mobile particles in $z<z_{\mathrm{e}}$. A match between an identified trajectory and a reference trajectory is considered successful if each coordinate of the tracking result falls within a $3 \times 3$ pixel window around the corresponding coordinate of the reference trajectory. The detection efficiency for deposited particles $\beta_{d}$ is defined as the number of successful matches between tracking results and reference locations of deposited particles divided by the number of deposited particles. The ratio of the number of invalid trajectories and the total number trajectories of mobile particles is represented by $\beta_{b}$.

Evaluation of 1000 image pairs gave a fraction of invalid matches of only $\beta_{b}=0.51 \%$. The tracking efficiency for deposited particles is $\beta_{d}=98.1 \%$. It may seem surprising that $\beta_{d}<$ $100 \%$, considering the simple pre-tracking procedure described above. However, in our computer generated particle images, particles were allowed to overlap and a small fraction of the deposited particles is expected to be too close to a neighboring particle in order to resolve the individual particle positions. Figure $6 a$ shows the tracking efficiency for mobile particles $\beta_{m}\left(z_{\mathrm{e}}\right)$. For $z_{\mathrm{e}}<10 \mu \mathrm{m}$, we find $\beta_{m}>90 \%$. The decreasing trend of $\beta_{m}$ in $2 \mu \mathrm{m}<z_{\mathrm{e}}<10 \mu \mathrm{m}$ is expected, as particles appear larger and less bright in an image the further away they are from the substrate. In other words, tracking of partially out-of-focus particles is more challenging 
than tracking of in-focus particles. The sudden change of the slope in Figure 6a around $z_{\mathrm{e}}=$ $10 \mu \mathrm{m}$ indicates that the relevant depth of field (after image processing) is close to $10 \mu \mathrm{m}$. Since the intensity distributions of individual particles in the synthetic images as a function of the particle distance from the object plane closely matches the corresponding particle image distribution of the experimental images [12], the depth of field in the experiments should also be close to $10 \mu \mathrm{m}$.

The precision of the detected location of particles is evaluated by taking the difference between the reference particle positions and the detected particle positions for all successfully matched trajectory pairs (tracking results and reference trajectory). The resulting differences in the horizontal components and in the vertical components of the particle image positions are all combined into one histogram for all trajectory pairs. For deposited particles (Figure $6 \mathrm{~b}$ and for mobile particles that are within $z<3 \mu \mathrm{m}$ (Figure $6 \mathrm{c}$ ), the precision is about $+/-0.1$ pixel $(+/-54.8 \mathrm{~nm})$ as expected [13]. For all valid trajectories of mobile particles, an overall precision of about $+/-0.25$ Pixel $(+/-137 \mathrm{~nm})$ is achieved (Figure $6 \mathrm{~d})$.

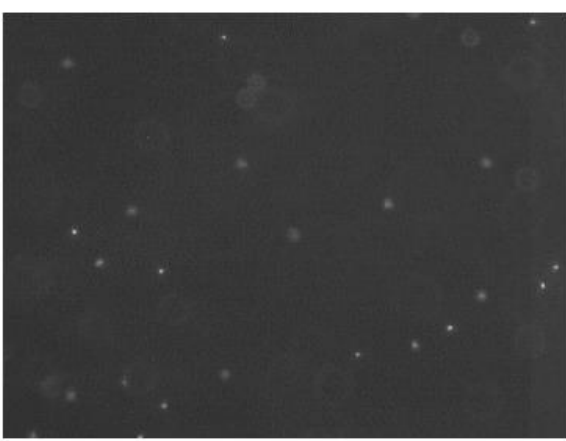

a)

b)

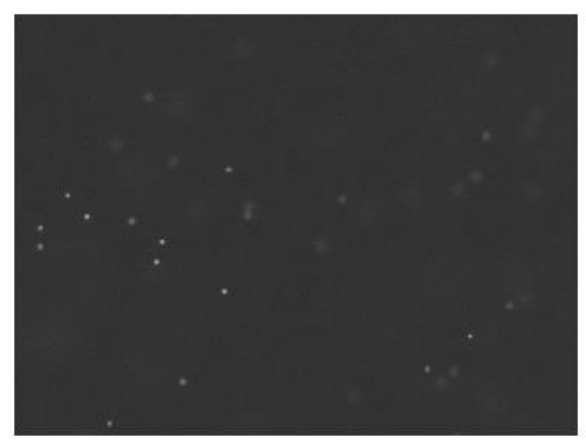

Figure 5: Excerpts of a particle image a) obtained from optical setup and b) corresponding computer generated image. Both images show the same field of view and consist of the same number of pixels and are based on the same particle concentration. Furthermore, both images are displayed by using the same color-map. 
a)

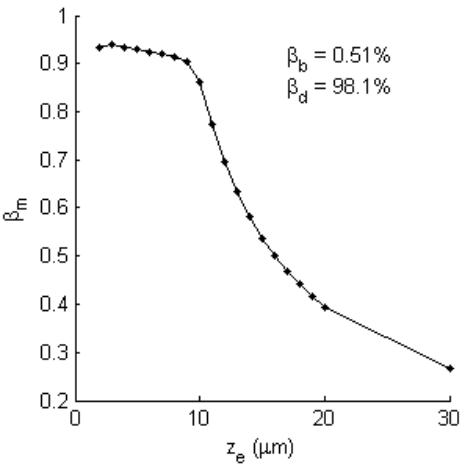

c)

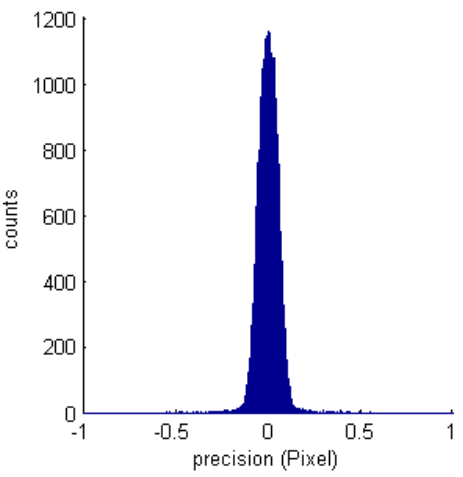

b)

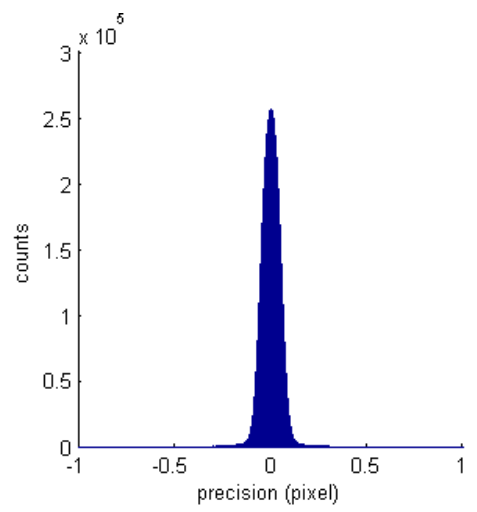

d)

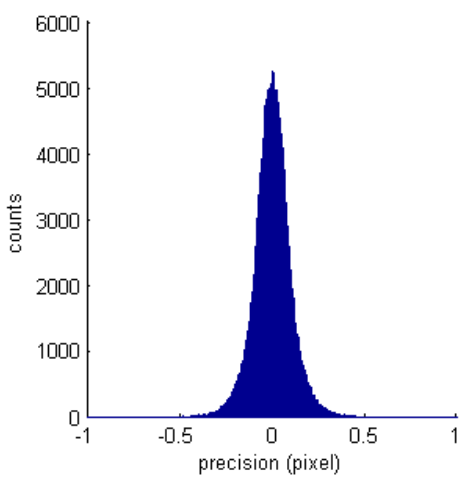

Figure 6: a) Tracking efficiencies $\beta_{m}\left(z_{\mathrm{e}}\right)$ and $\beta_{d}$ and fraction of invalid trajectories $\beta_{b}$; b) precision for deposited particles; c) precision valid trajectories of mobile particles within $z<3 \mu \mathrm{m}$; d) Precision for all valid trajectories

\section{$7 \quad$ Simulation method and parameters:}

In the notation of Weronski et al. the governing convective diffusion equation eq (5) of the main article after coordinate transformation reads

$$
\frac{\exp \left(2 x_{1}\right)}{F_{1}\left(x_{1}\right)} \frac{\partial y_{1}}{\partial \tau}=\frac{\partial^{2} y_{1}}{\partial x_{1}^{2}}+\frac{\partial y_{1}}{\partial x_{1}}\left(\frac{\partial y_{1}}{\partial x_{1}}+b_{1}\left(x_{1}\right)\right)+c_{1}\left(x_{1}\right)
$$

where $y_{1}=\ln (c)$ is the logarithm (naturalis) of the dimensionless particle concentration $c=n / n_{\mathrm{b}}$ with the bulk particle concentration $n_{\mathrm{b}}, \quad \tau=\frac{D_{0}}{a^{2}} t$ is the dimensionless time with the particle bulk diffusion coefficient $D_{0}$ and the particle radius $a$, and with the spatial variable

$$
x_{1}=\ln \left(H-H_{1}\right) \text {, }
$$

where $H=h / a$ is the non-dimensional particle-interface gap width and $\mathrm{H}_{1}=\mathrm{h}_{1}$ /a is the nondimensional gap width corresponding to the primary energy minimum. Expressions for the 
coefficients $\mathrm{F}_{1}\left(\mathrm{x}_{1}\right), \mathrm{b}_{1}\left(\mathrm{x}_{1}\right)$ and $\mathrm{c}_{1}\left(\mathrm{x}_{1}\right)$ in eq (4) can be found in [15]. Note that although Weronski et al. [15] only derived expressions for the steady state version of eq (4), the coefficient associated with the time derivative directly follows from the application of the coordinate transformation proposed by these authors. Eq (4) was solved by using the boundary conditions [15]

$$
\begin{gathered}
\lim _{x_{1} \rightarrow-\infty} \frac{\partial^{2} y_{1}}{\partial x_{1}^{2}}=0 \text { (perfect sink) } \\
\lim _{x_{1} \rightarrow \infty} y_{1}=0
\end{gathered}
$$

and the initial condition $y_{1}=0$ at $\tau=0$.

In general, equations (4)-(7) predict transient concentration profiles until a steady state concentration profile is reached after a certain time-period has passed [16], [17].

The boundary initial value problem described by eqs (4)-(7) (with the corresponding initial conditions) was solved for the logarithm of the dimensionless particle concentration by using the Matlab solver package pdepe. The lower and upper integration limits were set to $x_{1 \min }=$ $\ln \left(\mathrm{H}_{1}\right)-16$ and $x_{1 \max }=16.5000$ computational nodes were uniformly distributed between $\mathrm{x}_{1 \min }<$ $\mathrm{x}_{1}<2$ and 30 additional nodes were uniformly distributed over the remainder of the integration domain. The method's accuracy parameters were set to RelTol $=10^{-9}$ and AbsTol $=10^{-10}$. The transient solutions for the concentration fields were used in eq (11) together with the corresponding steady state concentration fields. All steady-state concentration fields and deposition rates were derived from solutions of the steady state version of eq (4) (i.e. with $\left.\frac{\partial y_{1}}{\partial \tau}=0\right)$. The steady state version of eq (4) was solved in two steps: First, eq (4) together with the perfect sink boundary condition eq (6) was solved for $\frac{\partial y_{1}}{\partial x_{1}}$ by using the Matlab solver ode15s. The method's accuracy parameters were set to RelTol $=3 \cdot 10^{14}$ and AbsTol $=10^{-15}$. Next, with known $\frac{\partial y_{1}}{\partial x_{1}}$, the logarithm of the dimensionless particle concentration $y_{1}$ was obtained by numerical integration based on the trapezoidal rule (Matlab function trapz) and the second boundary condition eq (7) was enforced. With known solutions for $c$ and $\frac{\partial c}{\partial H}$, the normal component of the particle flux was determined from eq (7) of the main article. In order to demonstrate that our implementation of the method was free of errors, Figure 4 of the original work [15] was reproduced as shown in 
Figure 7. Furthermore, all relevant simulation parameters used in this work, except for the zeta potentials and salt concentrations are listed in 


\section{Table 2.}

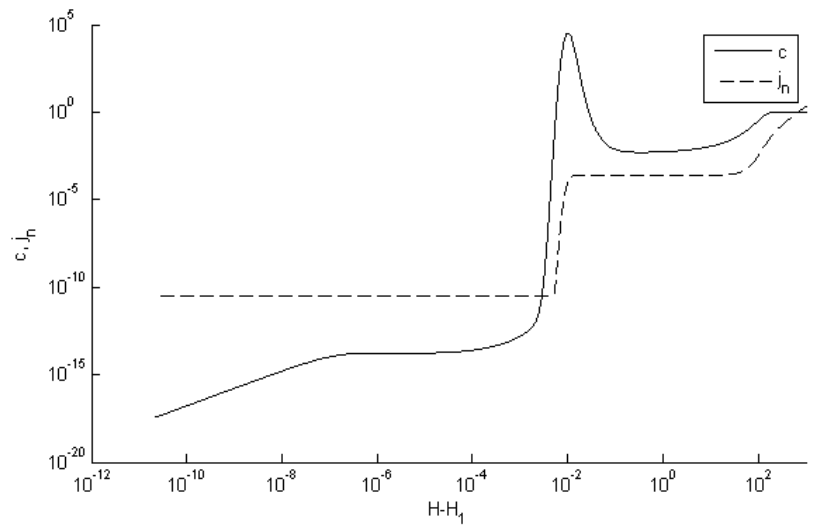

Figure 7: Figure 4 of [15] reproduced. $j_{n}$ is the dimensionless normal component of the particle flux as defined in [15]. 
Table 2: Simulation Parameters

\begin{tabular}{|c|c|c|}
\hline Gravity constant & $g$ & $9.81 \mathrm{~m} / \mathrm{s}^{2}$ \\
\hline Temperature & $\mathrm{T}$ & $295 \mathrm{~K}$ \\
\hline Particle radius & a & $500 \mathrm{~nm}$ \\
\hline Primary minimum distance & $\mathrm{h}_{1}$ & $0.158 \mathrm{~nm}$ \\
\hline Fluid density & $\rho_{\mathrm{f}}$ & $997 \mathrm{~kg} / \mathrm{m}^{3}$ \\
\hline Particle density & $\rho_{\mathrm{p}}$ & $1053 \mathrm{~kg} / \mathrm{m}^{3}$ \\
\hline Dynamic viscosity & $\eta$ & $0.958 \mathrm{mPas}$ \\
\hline Relative permittivity of water & $\varepsilon_{r}$ & 78.54 \\
\hline Reynolds number & $\mathrm{Re}$ & 0.75 \\
\hline Péclet number & $\mathrm{Pe}$ & $1.3 \cdot 10^{-4}$ \\
\hline Flow parameter & $\alpha_{\mathrm{r}}$ & 0.9975 \\
\hline Bulk diffusion coefficient & $\mathrm{D}_{0}$ & $0.485 \mu \mathrm{m}^{2} / \mathrm{s}$ \\
\hline Particle concentration & $\mathrm{n}_{\mathrm{B}}$ & $1.91 \cdot 10^{7} \mathrm{~cm}^{-3}$ \\
\hline Chamber inlet radius & $\mathrm{R}_{\mathrm{i}}$ & $1.1 \mathrm{~mm}$ \\
\hline Hamaker constant & $A_{132}$ & $1.54 \cdot 10^{-21} \mathrm{~J}$ \\
\hline Lower integration limit & $\mathrm{x}_{1 \min } ;\left(\mathrm{h}_{\min }\right)$ & $\ln \left(\mathrm{H}_{1}\right)-16 ;(1+\exp (-16)) \mathrm{h}_{1}$ \\
\hline Upper integration limit & $\mathrm{x}_{1 \max } ;\left(\mathrm{h}_{\max }\right)$ & $(\approx 4 \mathrm{~m})$ \\
\hline
\end{tabular}




\section{$8 \quad$ Transition to steady state conditions}

Figure 8 visualizes the expected transition to steady state concentration profiles after initialization of the flow at $t=0$ through the parameter $\Lambda$ as defined by eq 11 of the main article. These curves were computed by assuming the extended DLVO interaction profile eq 15 of the main article. Note that the curves for $C_{\mathrm{NaCl}}=100 \mathrm{mM}$ and $C_{\mathrm{NaCl}}=500 \mathrm{mM}$ were indistinguishable so that the curve obtained for $C_{\mathrm{NaCl}}=500 \mathrm{mM}$ was omitted from Figure 8. For experiments performed under unfavourable and favourable conditions, steady state conditions can be expected 15 minutes and 5 minutes after initialization of the flow, respectively. The same computation was performed by employing the DLVO interaction potential (main article eq 14) which predicts unfavourable conditions for salt concentrations $C_{\mathrm{NaCl}}<0.3 \mathrm{M}$. The DLVO theory predicts a significantly slower transition to steady state for $C_{\mathrm{NaCl}}=0.1 \mathrm{M}$ as compared to the extended DLVO theory. However, for all interaction potentials and salt concentrations considered in this work, a 15 minutes wait time from the time of initialization of the flow until initialization of image acquisition was found to be sufficient to achieve steady state conditions.

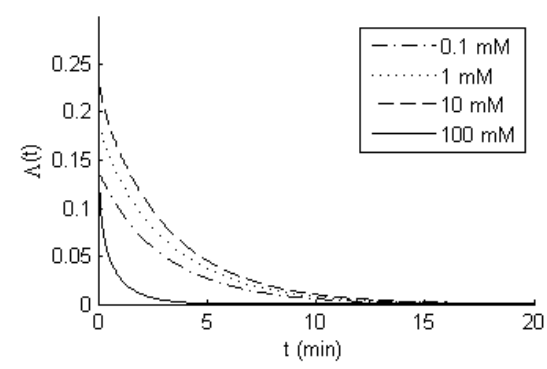

Figure 8: Transition to steady state concentration profiles for different salt concentrations with $\Lambda$ as defined in eq 11 (main article). The extended DLVO interaction potential was used for the computation.

\section{Effective diffusion constants of weakly immobilized particles and mean square displacement plots}

Section 3.2 of the main article examined drift velocities of weakly immobilized particles in Figure $4 b$ and $c$ (main article) and concluded that the lateral motion of these particles is hindered by non-hydrodynamic effects.

The same conclusion can be drawn from an analysis of the effective lateral diffusion coefficient

$$
D_{\mathrm{II} i}=\frac{\Delta X_{\mathrm{MSD} i}^{2}}{4 \Delta t}
$$


of the particles in Figure $4 \mathrm{~b}$ and $\mathrm{c}$ of the main article, which is estimated from the mean square displacement (MSD)

$$
\Delta X_{\mathrm{MSD} i}^{2}=\frac{1}{N} \sum_{j=1}^{N}\left|\Delta \vec{X}_{i}\left(t_{j}\right)\right|^{2}
$$

of the particles [13]. Of course, the particles in Figure $4 \mathrm{~b}$ and $c$ (main article) might not be undergoing free diffusion during the whole observation time, so that application of eq (9) on the entire trajectory data set would not be strictly meaningful. However, for the sake of the argument to be made here, eq (9) is applied to the entire trajectory data set and and eq (8) yields an effective diffusion coefficient. Also note, that the relatively small drift velocities of the particles has been neglected in the determination of the diffusion coefficient from eq (8) [18]. For $p=1 \mathrm{in} \mathrm{eq} \mathrm{(4)} \mathrm{(main} \mathrm{article)} \mathrm{so} \mathrm{that} \Delta t=10 \mathrm{~s}$ (and $N=N_{1} \approx 100$ ), this simplifying assumption is valid as diffusion dominates over drift on such a small timescale. The resulting effective lateral diffusion coefficients are $\frac{D_{\mathrm{II} 1}}{D_{0}}=0.0042$ and $\frac{D_{\mathrm{II} 2}}{D_{0}}=0.0026$ for the particle in Figure $4 \mathrm{~b}$ and $\mathrm{c}$, respectively, where $D_{0}=0.485 \frac{\mu \mathrm{m}^{2}}{\mathrm{~s}}$ is the diffusion coefficient of the particle in the bulk (as calculated from the Stokes Einstein equation). These values can be compared to well established predictions [17]

$$
\frac{D_{\mathrm{II}}}{D_{0}}=\frac{1}{K_{\mathrm{H} 2}}
$$

where $K_{\mathrm{H} 2}$ is a non-dimensional friction coefficient that accounts for hydrodynamic particle-wall interactions [19], [20]. However, similar to the particle drift velocity discussed above, such low values $\frac{D_{\mathrm{II}}}{D_{0}}$ would only be expected for particles that are much closer to the wall than the size of a water molecule [20]. Thus it can be concluded that the low observed effective lateral diffusion coefficients cannot be explained solely based on hydrodynamic particle-wall interactions.

Plots of the MSD over the time period $\Delta t$ can potentially provide further evidence on whether the lateral particle motion is hindered. Thus the MSD is calculated by setting $p=1,2,3$ in eq 4 of the main article so that $\Delta t=p \cdot 10 \mathrm{~s}$ and $N=N_{1}-p+1$ in eq (9). Figure $9 \mathrm{a}$ and b show the resulting plots of MSD over $\Delta t$ for the particles in Figure $4 \mathrm{~b}$ and $\mathrm{c}$ of the main article, respectively. In these plots, the error bars represent the expected standard deviation for the MSD based on the given number of samples in a trajectory [18]. A linear increase of the MSD with $\Delta t$ indicates free diffusion of the particles [21]. If the particles are trapped, i.e. in a potential well, the MSD is expected to reach a limiting value for large $\Delta t$ [21]. Although the trends of the 
MSD over $\Delta t$ would support the hypothesis of confined lateral particle motion, the high statistical uncertainty for the MSD at large $\Delta t$ due to the relatively low number of samples for each trajectory does not allow drawing this conclusion [18].

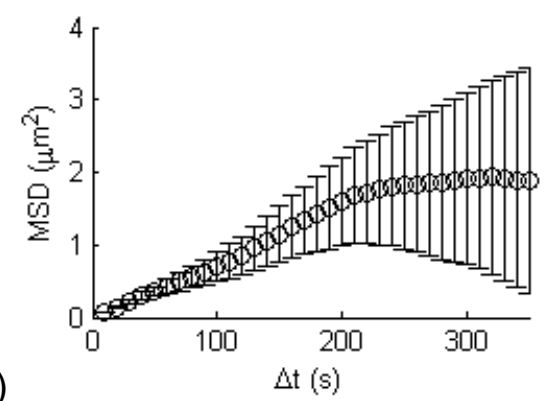

a)

Figure 9: Mean square displacement (MSD) of a particle as a function of the time interval $\Delta t$. The error bars represent the expected standard deviation of the MSD based on the given sample size and $\Delta t$ [72]. a) and b) show MSD-plots for the particles in Figure 4b and c of the main article, respectively.

\section{Statistical analysis of a monolayer coverage}

Figure 10 shows particle images that were acquired at different times during a deposition experiment at unfavourable conditions for deposition (sodium chloride concentration of $C_{\mathrm{NaCl}}=10 \mathrm{mM}$ ). As discussed in section 3.3 of the main article, particle deposition at a salt concentration of $C_{\mathrm{NaCl}}=10 \mathrm{mM}$ is expected to be governed by surface heterogeneity. In other words, particles can only deposit on certain regions of the substrate where repulsive surface interactions vanish due to local surface roughness or low local surface charge density.

If these "favorable" regions are large enough relative to the field of view, one may be able to assess the degree of surface heterogeneity by comparing the distribution of the deposited particles in Figure 10c to a Poisson distribution

$$
f_{\text {pois }}\left(n_{\mathrm{p}}\right)=\frac{\lambda^{n_{\mathrm{p}}}}{n_{\mathrm{p}} !} \exp (-\lambda)
$$

where $n_{\mathrm{p}}$ is the number of deposited particles in a small sub-region of the entire field of view in Figure 10c and

$$
\lambda=\frac{n_{\text {pall }}}{A_{\text {FoV }}} A_{\text {sub }}
$$


is the expected number of particles in a sub-region with the sub-region area $A_{\text {sub, }}$ the total number of deposited particles $n_{\text {pall }}=204$ and with the area of the entire field of view $A_{\mathrm{FoV}}=$ $658 \mu \mathrm{m} \cdot 877 \mu \mathrm{m}$. Near the flow stagnation point $\left(r / R_{\mathrm{i}}<0.5\right.$ at moderate Reynolds number) the distribution of deposited particles is expected to follow a Poisson distribution given that the substrate surface is homogeneous. Deviations of the actual particle distribution from the Poisson distribution may provide valuable information about the heterogeneity of the substrate.

Hence, the entire field of view in Figure 10c was subdivided into non-overlapping quadratic sub-regions with area $A_{\text {sub }}$. Counts $n_{\mathrm{p}}$ of deposited particles in each of the sub-regions were determined by using a Matlab script. Next, this information was combined into a discrete probability density function $f_{\text {rel }}\left(n_{\mathrm{p}}\right)$, which represents the number of sub-regions with $n_{\mathrm{p}}$ deposited particles divided by the total number of sub-regions $N_{\text {sub }}=A_{\text {Fov }} / A_{\text {sub }}$.

Figure 11 shows $f_{\text {rel }}$ and $f_{\text {pois }}$ corresponding to the deposited particles in Figure 10c for three sub-region sizes $A_{\text {sub. }}$. While $f_{\text {rel }}$ and $f_{\text {pois }}$ match well for the smallest sub-region area (Figure $11 \mathrm{a}, A_{\text {sub }}=50$ pixel $\times 50$ pixel), $f_{\text {rel }}$ differs significantly from $f_{\text {pois }}$ for the two larger sub-region sizes in Figure $11 \mathrm{~b}$ and $\mathrm{c}$. However, the deviation of $f_{\text {rel }}$ from $f_{\text {pois }}$ cannot easily be attributed to the heterogeneous nature of the substrate surface. I.e., with increasing sub-region size $A_{\text {sub, }}$ the number of available samples $N_{\text {sub }}=A_{\text {Fov }} / A_{\text {sub }}$ from which $f_{\text {rel }}$ is derived decreases and the deviation of $f_{\text {rel }}$ from $f_{\text {pois }}$ may simply be a result of the uncertainty of $f_{\text {rel }}$ due to low number of available samples at the larger sub-region sizes in Figure $11 \mathrm{~b}$ and c.

This can be demonstrated by a simple numerical experiment where $n_{\text {pall }}=204$ deposited particles are randomly distributed across a virtual region with area $A_{\mathrm{Fov}}$ before a discrete probability density function $f^{\text {num }}\left(n_{\mathrm{p}}\right)$ is derived from the resulting computer generated particle distribution by using the same method that was used to derive $f_{\text {rel }}$ from the experimental particle distributions. This procedure is repeated $N_{v}=5000$ times in order to obtain many computer generated probability density distributions $f_{i}^{\text {num }}\left(n_{\mathrm{p}}\right)$ with $i=1, \ldots, 5000$. The average

$$
f_{\text {pois }}^{\text {num }}\left(n_{\mathrm{p}}\right)=\frac{\sum_{i=1}^{N_{\mathrm{v}}} f_{i}^{\text {num }}\left(n_{\mathrm{p}}\right)}{N_{\mathrm{v}}} \approx f_{\text {pois }}\left(n_{\mathrm{p}}\right)
$$

of all numerically generated probability density distributions $f_{i}^{\text {num }}$ is approximately equal to the Poisson distribution $f_{\text {pois }}$ in equations (11)-(12) as it can be seen in Figure 11. The error bars in Figure 11 represent the two times the corresponding standard deviation of the computer generated probability density distributions $f_{i}^{\text {num }}$. In general, all open circles in Figure 11a-c fall 
within the errorbars which suggests that the deviation of $f_{\text {rel }}$ and $f_{\text {pois }}$ may simply be a result of the uncertainty in $f_{\text {rel }}$ due to the low number of available samples used to generate $f_{\text {rel }}$. One exception can be seen in Figure 11c, where $f_{\text {rel }}\left(n_{\mathrm{p}}=11\right)$ clearly deviates from $f_{\text {pois }}$. We believe that this anomaly is a result of 3 deposited small particle aggregates in Figure 10c.

It can be concluded that the statistical analysis of the particle distributions performed in this sub-section does not provide any valuable insight into the heterogeneity of the substrate surface. 


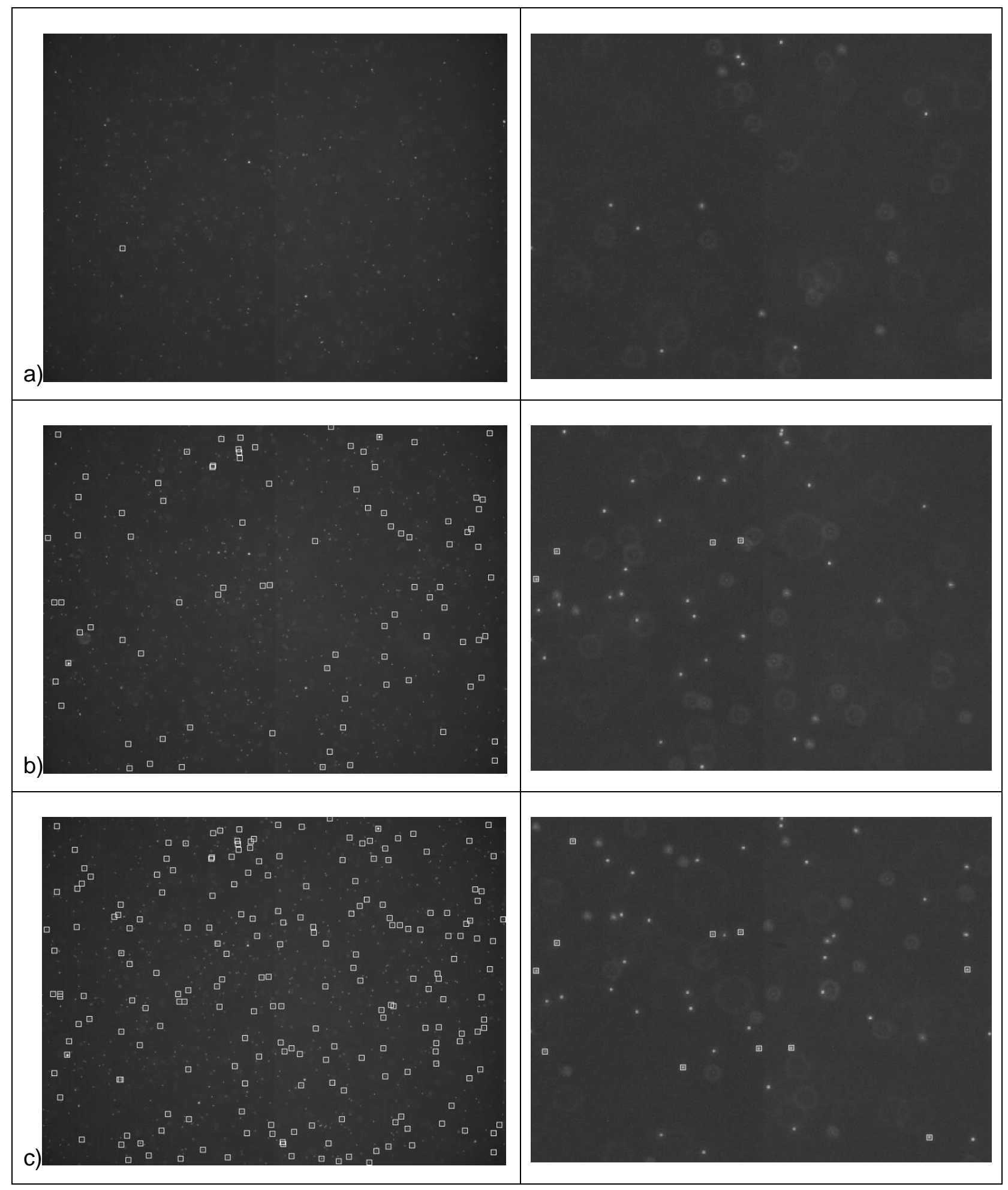

Figure 10: Particle images acquired at different times during a deposition experiment at a sodium chloride concentration of $C_{\mathrm{NaCl}}=10 \mathrm{mM}$ : a) $\left.\left.\mathrm{t}=20 \mathrm{~s}, \mathrm{~b}\right) \mathrm{t}=500 \mathrm{~s}, \mathrm{c}\right)=1000 \mathrm{~s}$. The left sides in a-c show the full field of view $(658 \mu \mathrm{m} \times 877 \mu \mathrm{m}$, centered on the flow stagnation point). The right sides in a-c show close up views in a rectangular region $(164 \mu \mathrm{m} \times 218 \mu \mathrm{m})$ near the flow stagnation point. Particles that deposited after $t=10 \mathrm{~s}$ are surrounded by white squares. 


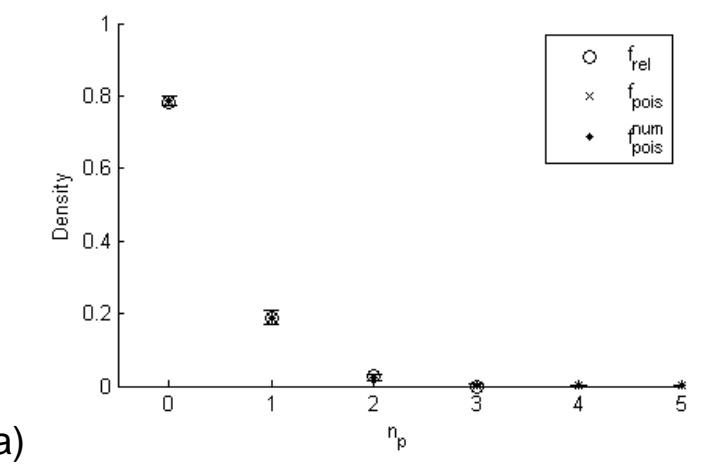

b)
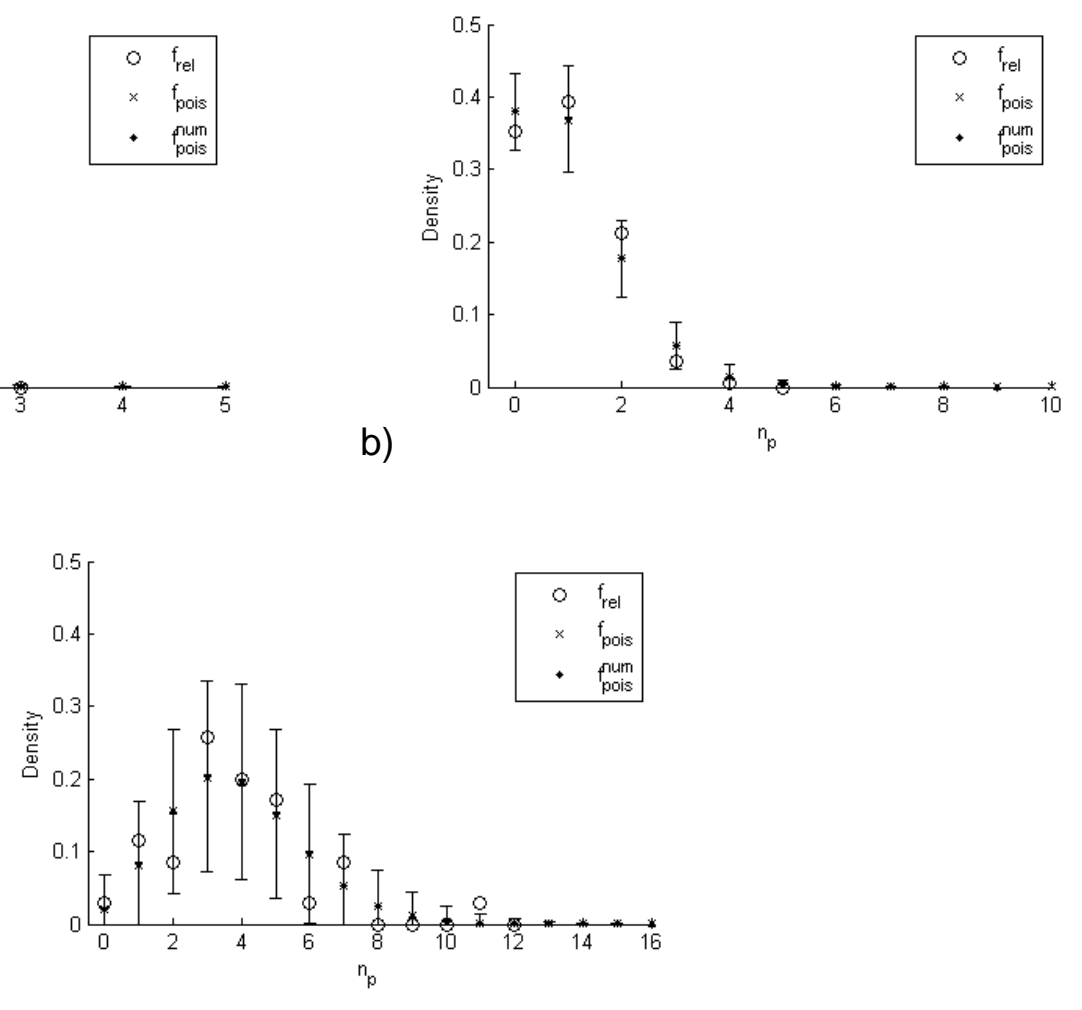

Figure 11: Distribution of deposited particles in Figure $10 \mathrm{c}$ for three different sub-region sizes a) $A_{\text {sub }}=50$ pixel $\times 50$ pixel, b) $A_{\text {sub }}=100$ pixel x 100 pixel and c) $A_{\text {sub }}=200$ pixel $\times 200$ pixel

\section{Surface energy data and Hamaker constants}

Some (by far not all) values for the Hamaker constants $A_{i i}$ that have been reported or used in literature are compiled in Table 3. Depending on the choice of the values for $A_{i i}$, Hamaker constants between $A_{132}=-1.4 \cdot 10^{-21} \mathrm{~J}$ and $A_{132}=1.9 \cdot 10^{-21} \mathrm{~J}$ can be obtained, where the limits correspond to either weak repulsive or weak attractive van der Waals interaction, respectively. A significantly larger value of the Hamaker constant $\left(A_{132}=5.2 \cdot 10^{-20} \mathrm{~J}\right)$ for the polystyrene-water-(Sylgard 184) PDMS system has been determined from direct force measurements between a polystyrene microsphere and a PDMS substrate in aqueous saline solution by using an atomic force microscope [22]. We conclude that severe discrepancies exist between different reported values for the Hamaker constant $A_{132}$ for the polystyrene-waterPDMS system. Until more accurate data is available we simply choose some of the more commonly used values for the Hamaker constants, i.e. $A_{11}=7.9 \cdot 10^{-20} \mathrm{~J}$ (for polystyrene) [23], [24], $A_{22}=4.4 \cdot 10^{-20} \mathrm{~J}$ (for PDMS) [25], [26], [27], and $A_{33}=3.7 \cdot 10^{-20} \mathrm{~J}$ (for water) [28], [23], [26], [29], [30]. These choices yield a Hamaker constant for the polystyrene-water-PDMS system of $A_{132}=1.5 \cdot 10^{-21} \mathrm{~J}$, which indicates rather weak attractive van der Waals forces. This particular choice for the Hamaker constant supports the discussion of our experimental results 
in the main article. Admittedly, the chosen value may seem arbitrary considering the large range of reported values. Thus, we discuss implications of other choices for $A_{132}$ on the conclusions of this work in the supporting information (section 13).

Table 3: Surface energy data. All surface energies are in $\mathrm{mJ} / \mathrm{m}^{2}$, the unretarded Hamaker constants $A_{i i}$ are displayed in $10^{-20} \mathrm{~J}$.

\begin{tabular}{|l|c|c|c|l|}
\hline Material & $A_{\mathrm{ii}}$ & $\gamma_{i}^{+}$ & \multicolumn{1}{|c|}{$\gamma_{i}^{-}$} & Reference \\
\hline PDMS & 4.3 & 0 & 3.05 & {$[24]$} \\
\hline PDMS & 3.5 & 0.64 & 0.32 & {$[31]$} \\
\hline PDMS & 4.4 & - & - & {$[25][26][27]$} \\
\hline PDMS & 4.5 & - & - & {$[29][32]$} \\
\hline PS & 7.9 & 0.08 & 0.15 & {$[24]$} \\
\hline PS & 8.3 & 0.46 & 2.22 & {$[33]$} \\
\hline PS & 8 & 1.3 & 3.1 & {$[34]$} \\
\hline PS & 7.1 & 0.57 & 5.27 & {$[35]$} \\
\hline PS & $6.6-7.9$ & & & {$[23]$} \\
\hline water & 4.1 & 25.5 & 25.5 & {$[36][31][24][33][34][35]$} \\
\hline water & 3.7 & - & - & {$[28][23][26][29][30]$} \\
\hline
\end{tabular}

\section{On the choice of the decay length $\lambda_{0}$ for the acid-base interactions}

Assigning an appropriate value for the decay length $\lambda_{0}$ ideally requires the direct measurement of the interaction forces for the given material combination by using a surface force apparatus [23] or atomic force microscope [37]. According to van Oss [36], appropriate values are in the range $0.6 \mathrm{~nm}<\lambda_{0}<1 \mathrm{~nm}$ and Israelachvili et al. suggests $0.3 \mathrm{~nm}<\lambda_{0}<2 \mathrm{~nm}$ [38] while specifically reporting $\lambda_{0}=1.6 \mathrm{~nm}$ for the interaction of two (un-crosslinked) PDMS monolayers. 
Note, that much larger decay lengths can be observed for the interaction of hydrophobic substrates when the fluids have not been degassed [38]. Newby et al. [24] used $\lambda_{0}=0.6 \mathrm{~nm}$ to model the $A B$-interaction between bovine serum albumin and crosslinked PDMS. In the current work, we arbitrarily set $\lambda_{0}=1 \mathrm{~nm}$.

\section{Implications of other choices for the Hamaker constant $\boldsymbol{A}_{132}$}

Most of the discussion of the results in the main article depends to a strong degree on the selected value for the Hamaker constant. It seems mandatory to challenge the conclusions of this work by considering other values for the Hamaker constant in the range of the reported values. First of all, we rule out the negative Hamaker constants that can be obtained by combining the Hamaker constant for PDMS by [31] with any of the other reported values through equation 21. All other combinations indicate attractive van der Waals forces which are, in our opinion, more in line with the observed particle deposition events. In the following discussion, we consider three choices for the Hamaker constants: $A_{132}^{(\min )}=2.8 \cdot 10^{-22} \mathrm{~J}$ is the smallest (positive) constant that can be obtained by combining values listed in Table 3 through equation $21, A_{132}=1.5 \cdot 10^{-21} \mathrm{~J}$ is the value that was chosen for this work and $A_{132}^{(\max )}=5.2$. $10^{-20} \mathrm{~J}$ is the largest reported value that we found. Table 4 lists minimum separation distances $H_{0}$ and expected number of mobile particles $N_{\text {pe }}$ in the field of view that are obtained by employing these Hamaker constants in combination with the DLVO theory and extended DLVO theory. Particle counts in the range $55 \leq N_{\text {pe }} \leq 58$ and the minimum separation distance $H_{0}=0.0003$ indicate favourable conditions for deposition and $N_{\text {pe }}>60$ and $H_{0}>0.0003$ indicate unfavourable conditions for deposition. All fields in Table 4 corresponding to favourable conditions for deposition are highlighted in gray. We believe, that the experimental conditions for deposition were favourable at the largest two salt concentrations $\left(C_{\mathrm{NaCl}}=0.1 \mathrm{M}\right.$ and $\left.0.5 \mathrm{M}\right)$ as the measured particle counts $N_{\text {pexp }}$ at these salt concentrations were similar and significantly lower than $N_{\text {pexp }}$ at lower salt concentrations $C_{\mathrm{NaCl}}<0.1 \mathrm{M}$ and the measured deposition rates matched the predicted mass transfer rates reasonably well. We begin the discussion by considering $A_{132}^{(\max )}$. According to Table 4, both DLVO and extended DLVO theory seem to capture the transition from favourable to unfavourable deposition and additional measurements at salt concentrations between $10 \mathrm{mM}$ and $100 \mathrm{mM}$ would have to be conducted in order to favour one over the other. However, with $A_{132}^{(\max )}$, both DLVO and xDLVO predict significant accumulation $\left(N_{\text {pe }}=498\right)$ of mobile particles due to deep secondary minima at $C_{\mathrm{NaCl}}=10 \mathrm{mM}$. Comparison of the trends $N_{\text {pe }}$ and $N_{\text {pexp }}$ with respect to the salt 
concentration $C_{\mathrm{NaCl}}$ suggests that there was no deep secondary minimum during the experiments as the measured particle counts for unfavourable conditions increase more gradually with the salt concentration as compared to the expected counts.

Let us now consider $A_{132}^{(\mathrm{min})}$. From Table 4 it can be seen that the DLVO theory does not capture the observed transition from unfavourable to favourable conditions when $A_{132}^{(\min )}$ is chosen. The extended DLVO theory on the other hand does not allow us to favour the chosen $A_{132}$ over $A_{132}^{(\min )}$ with the available experimental data as the corresponding trends of $H_{0}$ and $N_{\text {pe }}$ with respect to $C_{\mathrm{NaCl}}$ are nearly identical. However, in section 3.4 we suggest that the relatively larger fraction $\Upsilon$ of slow particles at $C_{\mathrm{NaCl}}=1 \mathrm{mM}$ and $10 \mathrm{mM}$ may be explained by shallow secondary minima that make particle escape into the bulk less likely at these salt concentrations. With $A_{132}^{(\mathrm{min})}$, the extended DLVO interaction potentials do not exhibit a secondary minimum at any of the investigated salt concentrations. Thus it can be concluded that overall, a Hamaker constant close to $A_{132}=1.5 \cdot 10^{-21} \mathrm{~J}$ seem to allow the most plausible interpretation of the experiments performed in line with this work.

Table 4: Minimum (dimensionless) separation distances $H_{0}$ and expected particle counts $N_{\text {pe }}$ within the field of view obtained by employing the DLVO theory and the extended DLVO theory in combination with different Hamaker constants and for different salt concentrations $C_{\mathrm{NaCl}}$

\begin{tabular}{|c|c|c|c|c|c|c|c|c|c|c|c|c|c|}
\hline \multirow{3}{*}{$\begin{array}{l}C_{\mathrm{NaCl}} \\
(\mathrm{mM})\end{array}$} & \multicolumn{6}{|l|}{ DLVO } & \multicolumn{6}{|c|}{ Extended DLVO } & \multirow[t]{3}{*}{$N_{\text {pexp }}$} \\
\hline & \multicolumn{2}{|c|}{$A_{132}^{(\min )}$} & \multicolumn{2}{|c|}{$A_{132}$} & \multicolumn{2}{|c|}{$A_{132}^{(\max )}$} & \multicolumn{2}{|c|}{$A_{132}^{(\min )}$} & \multicolumn{2}{|c|}{$A_{132}$} & \multicolumn{2}{|c|}{$A_{132}^{(\max )}$} & \\
\hline & $H_{0}$ & $N_{\mathrm{pe}}$ & $H_{0}$ & $N_{\mathrm{pe}}$ & $H_{0}$ & $N_{\mathrm{pe}}$ & $H_{0}$ & $N_{\mathrm{pe}}$ & $H_{0}$ & $N_{\mathrm{pe}}$ & $H_{0}$ & $N_{\mathrm{pe}}$ & \\
\hline 0.1 & 0.3267 & 110 & 0.3267 & 110 & 0.3263 & 110 & 0.3267 & 110 & 0.3271 & 110 & 0.3262 & 111 & 164 \\
\hline 1 & 0.1027 & 112 & 0.1027 & 112 & 0.1007 & 113 & 0.1027 & 112 & 0.1027 & 112 & 0.1007 & 113 & 220 \\
\hline 10 & 0.0301 & 113 & 0.03 & 113 & 0.0253 & 498 & 0.0301 & 113 & 0.0299 & 113 & 0.0253 & 498 & 263 \\
\hline 100 & 0.0065 & 113 & 0.0068 & 114 & 0.0003 & 55 & 0.0003 & 58 & 0.0003 & 58 & 0.0003 & 55 & 81 \\
\hline 500 & 0.0003 & 60 & 0.0003 & 58 & 0.0003 & 55 & 0.0003 & 58 & 0.0003 & 58 & 0.0003 & 55 & 90 \\
\hline
\end{tabular}




\section{Displacement distributions with standard deviation}

a)
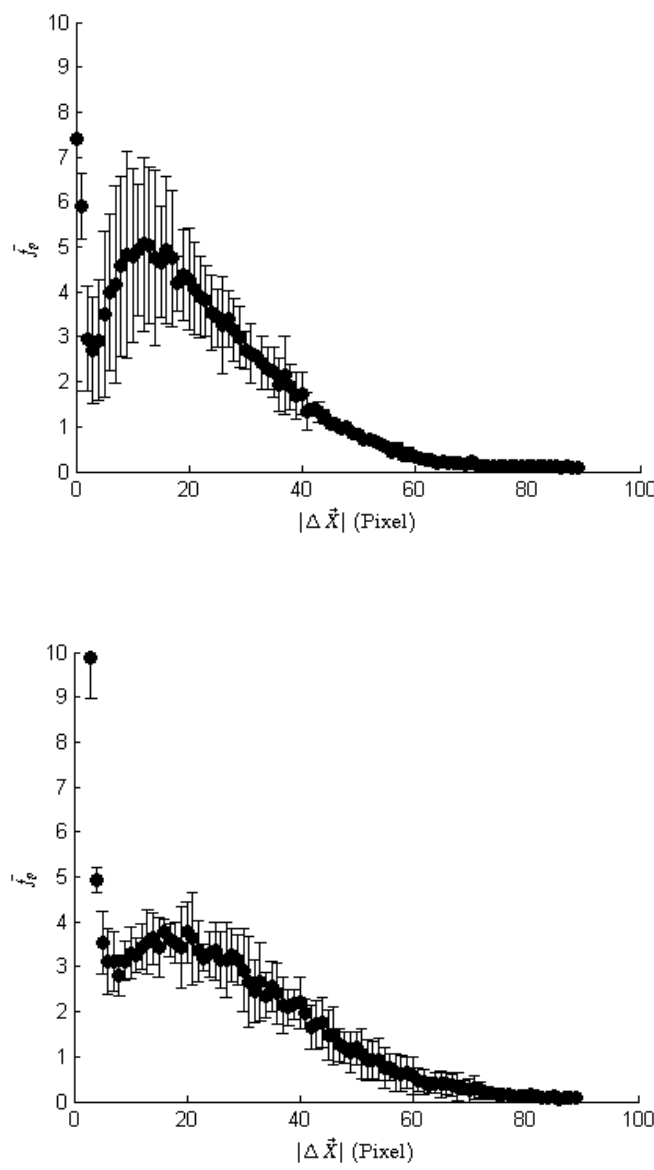

b)

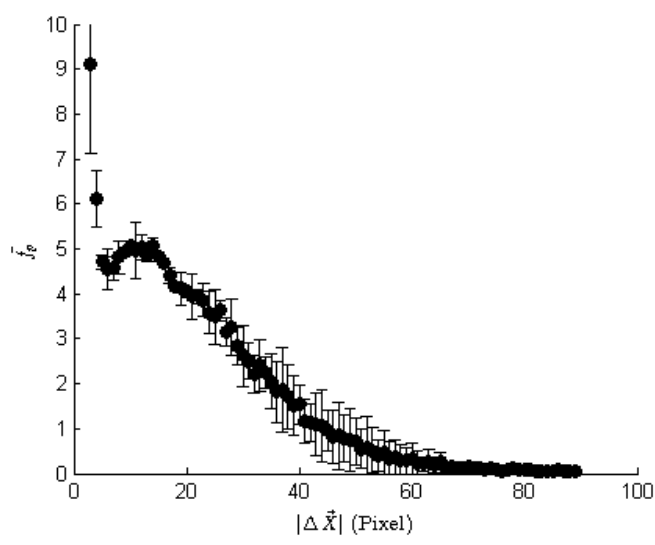

d)

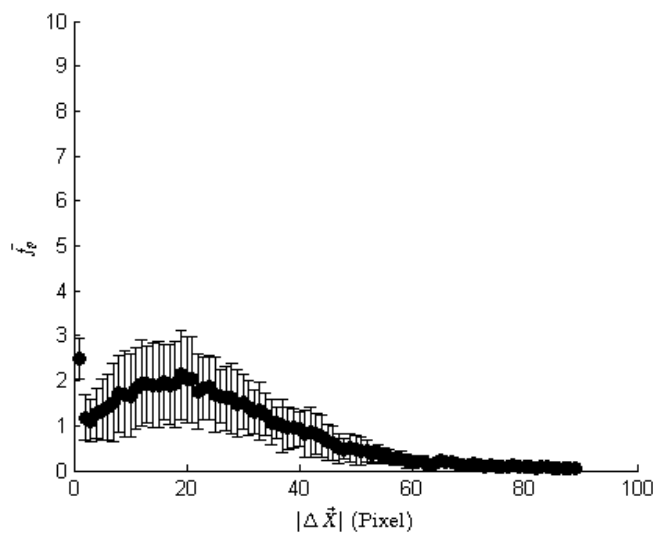

Figure 12: Average displacement distributions from Figure 7a) (main article) with associated standard deviation shown as error bars for different salt concentrations $C_{\mathrm{NaCl}}$ : a) $0.1 \mathrm{mM}$, b) $1 \mathrm{mM}$, c) $10 \mathrm{mM}, \mathrm{d}$ ) $100 \mathrm{mM}$.

\section{Model for displacement distributions}

A simple numerical model was developed in order to estimate the expected displacement distributions for the case where particles are not weakly immobilized. A large number of virtual particles are distributed in a virtual region above the substrate. We then assign an expected displacement after $\Delta t=10$ seconds to each particle only based on its initial position in the flow field and with respect to the substrate while completely neglecting any dynamical aspects occurring within the $\Delta t=10$ seconds. Finally, the expected displacements are combined into histograms for qualitative comparison with the histograms obtained from the laboratory experiments. In order to distribute virtual particles in the model domain $x$ - and $y$ - coordinates (in the plane parallel to the substrate) were generated by using the Matlab function rand to achieve 
a random distribution at the desired concentration. The expected non-uniform concentration profiles in the direction perpendicular to the substrate can be taken into account by sampling the z-positions from a probability density function (pdf) that is directly proportional to the concentration profiles. This is achieved in approximate manner by dividing the region $z<10 \mu \mathrm{m}$ of interest above the substrates into 1000 small sub-slices with thickness $\Delta z_{\mathrm{s}}$. The planes delimiting the $i$ th slice are denoted by $z_{\mathrm{b} i}=a+H_{0} a+(i-1) \Delta z_{S}$ and $z_{\mathrm{e} i}=a+H_{0} a+i \Delta z_{S}$ and the center of the slice is at $z_{\mathrm{c} i}=a+H_{0} a+\left(i-\frac{1}{2}\right) \Delta z_{s}$, where $H_{0}$ is the minimum separation distance as defined in section 3.1.2 (main article) (and listed in Table 2 of the main article). In each slice, a large number $N_{\mathrm{s} i}=2000 c\left(z_{\mathrm{c} i}\right)$ of particle positions are generated to achieve the corresponding distributions. As a result, the concentration of virtual particles was close to the predicted particle concentration everywhere in the model domain. Let the positions of the virtual particles be denoted as $\vec{X}_{\mathrm{v} i}=\left[x_{\mathrm{v} i} y_{\mathrm{v} i}\right]$ and $z_{\mathrm{v} i}$. Next, the radial flow velocity eq 2 (main article) is evaluated at each virtual particle position and transformed into Cartesian coordinates $v_{\mathrm{vx} i}, v_{\mathrm{vy} i}$. For each particle, the expected displacement after $\Delta t=10$ seconds is defined as

$$
\left|\Delta \vec{X}_{\mathrm{v}}\right|_{i}=\sqrt{\left(K_{\mathrm{H} 1}\left(h_{\mathrm{v} i}\right) v_{\mathrm{vx} i} \Delta t+\Delta x_{\mathrm{D} i}\right)^{2}+\left(K_{\mathrm{H} 1}\left(h_{\mathrm{v} i}\right) v_{\mathrm{vy} i} \Delta t+\Delta y_{\mathrm{D} i}\right)^{2}}
$$

where $K_{\mathrm{H}}\left(h_{\mathrm{v} i}\right)$ is a dimensionless hydrodynamic correction factor accounting for hydrodynamic particle-wall interactions, $h_{\mathrm{v} i}=z_{\mathrm{v} i}-a$ is the separation distance of the particle from the wall and $\Delta x_{\mathrm{D} i}$ and $\Delta y_{\mathrm{D} i}$ are random displacements due to Brownian motion. The random displacements were sampled from normal distributions with zero mean and a standard deviation of

$$
\sigma_{\mathrm{D} i}=\sqrt{\frac{2 D_{0} \Delta t}{K_{\mathrm{H} 2}\left(h_{\mathrm{v} i}\right)}}
$$

where $K_{\mathrm{H} 2}\left(h_{\mathrm{v} i}\right)$ is a hydrodynamic correction factor. Values for the correction factors $K_{\mathrm{H} 1}$ and $K_{\mathrm{H} 2}$ were interpolated from numerical data provided by [39] and [19], respectively.

\section{On the effect of gravity}

Considering the relatively small particle size of $1 \mu \mathrm{m}$ and the small difference between the density of the particle material and fluid, it may seem surprising that the discussion in section 3.1.2 of the main article suggests that gravity has a significant influence on the particle concentration profiles. Here we would like to demonstrate the significance of gravity in our 
experiments by a simple analysis where we compare the well-known Levich approximation for the particle adsorption flux

$$
j_{\text {Levich }}=0.78 \frac{n_{b} \alpha_{0}^{1 / 3} V_{\infty}^{1 / 3} D_{0}^{2 / 3}}{R_{\mathrm{i}}^{2 / 3}}
$$

(see e.g. [40]) to the particle flux

$$
j_{\text {grav }}=n_{b} \frac{2 a^{2}}{9 \eta} g\left(\rho_{\mathrm{f}}-\rho_{\mathrm{p}}\right)
$$

due to gravity in an unbounded quiescent dilute suspension through a plane that is perpendicular to the gravity vector. Note, that the Levich approximation neglects non-specific surface interactions, external forces and hydrodynamic particle-wall interactions. Although these two fluxes are technically not additive, we believe that the ratio $j_{\text {grav }} / j_{\text {Levich }}$ is still a good indicator for the relative importance of gravity on the deposition rates. Entering the values from

Table 2 along with $V_{\infty}=658 \frac{\mu m}{s}$ into eqs (16)-(17) gives $\frac{j_{\text {grav }}}{j_{\text {Levich }}} \approx 80 \%$, which suggests that gravity is an important driving force for deposition in our experiments.

\section{Bibliography}

[1] T. R. Simpson, Z. Tabatabaian, C. Jeynes, B. Parbhoo and J. L. Keddie, "Influence of Interfaces on the Rates of Crosslinking in," Journal of Polymer Science: Part A: Polymer Chemistry, vol. 42, pp. 1421-1451, 2004.

[2] G. C. Randall and P. S. Doyle, "Permeation-driven flow in poly(dimethylsiloxane)," PNAS, vol. 102, no. 31, p. 10813-10818, 2005.

[3] M. W. Toepke and D. J. Beebe, "PDMS absorption of small molecules and consequences in microfluidic applications," Lab on a Chip, vol. 6, p. 1484-1486, 2006.

[4] J. Flueckiger, V. Bazargan, B. Stoeber and K. C. Cheung, "Characterization of postfabricated parylene C coatings inside PDMS microdevices," Sensors and Actuators B: Chemical, vol. 160, pp. 864-874, 2011.

[5] H. Ohshima, T. W. Healy and L. R. White, "Approximate analytic expressions for the 
electrophoretic mobility of spherical colloidal particles and the conductivity of their dilute suspensions," Journal of the Chemical Society, Faraday Transactions 2: Molecular and Chemical Physics, vol. 79, no. 11, pp. 1613-1628, 1983.

[6] B. J. Kirby and E. F. Hasselbrink Jr, "Zeta potential of microfluidic substrates: 2. Data for polymers," Electrophoresis, vol. 25, p. 203-213, 2004.

[7] B. J. Kirby and E. F. Hasselbrink, "Zeta Potential of microfluidic substrates: 1. Theory, experimental techniques, and effects on separations," Electrophoresis, vol. 25, pp. 187212, 2004.

[8] W. Zeng, I. Jacobi, D. J. Beck, S. Li and H. A. Stone, "Characterization of syringe-pumpdriven induced pressure fluctuations in elastic microchannels," Lab on a Chip, vol. 15, no. 4, pp. 1110-1115, 2015.

[9] C. Gomez Suarez, J. Noordmans, H. C. van der Mei and H. J. Buscher, "Removal of Colloidal Particles from Quartz Collector," Langmuir, vol. 15, pp. 5123-5127, 1999.

[10] P. Sharma, M. Flury and J. Zhou, "Detachment of colloids from a solid surface by a moving air-water interface," Journal of Colloid and Interface Science, vol. 326, p. 143-150, 2008.

[11] V. Lazouskaya, L.-P. Wang, D. Or, G. Wang, J. L. Caplan and Y. Jin, "Colloid mobilization by fluid displacement fronts in channels," Journal of Colloid and Interface Science, vol. 406, pp. 44-50, 2013.

[12] B. Mustin and B. Stoeber, "Effect of linear image processing on the depth of correlation in micro PIV," Experiments in fluids, vol. 55:1817, 2014.

[13] J. C. Crocker and D. G. Grier, "Methods of Digital Video Microscopy for Colloidal Studies," Journal of Colloid and Interface Science, vol. 179, p. 298-310, 1996.

[14] M. Pilu, "A direct method for stereo correspondence based on singular value decomposition," in Conference on Computer Vision and Pattern Recognition, 1997. Proceedings., 1997 IEEE Computer Society, San Juan, 1997.

[15] P. Weronski and M. Elimelech, "Novel numerical method for calculating initial flux of colloid particle," Journal of Colloid and Interface Science, vol. 319, pp. 406-415, 2008. 
[16] M. Elimelech, J. Gregory, X. Jia and R. A. Williams, Particle Deposition \& Aggregation Measurement, Modelling and Simulation, Elsevier, 1995.

[17] Z. Adamczyk, Particles at Interfaces, Interactions, Deposition, Structure, Academic Press, 2006.

[18] H. Qian, M. P. Sheetz and E. L. Elson, "Single particle tracking. Analysis of diffusion and flow in two-dimensional systems," Biophys J., vol. 60, no. 4, p. 910-921, 1991.

[19] M. E. O'Neill, "A Slow motion of viscous liquid caused by a slowly moving solid sphere," Mathematika, vol. 11, no. 1, pp. 67- 74, 1964.

[20] A. J. Goldman, R. G. Cox and H. Brenner, "Slow viscous motion of a sphere parallel to a plane wall—I Motion through a quiescent fluid," Chemical Engineering Science, vol. 22, no. 4, p. 637-651, 1967.

[21] N. Ruthardt, D. C. Lamb and C. Braeuchle, "Single-particle Tracking as a Quantitative Microscopy-based Approach to Unravel Cell Entry Mechanisms of Viruses and Pharmaceutical Nanoparticles," Molecular Therapy, vol. 19, no. 7, p. 1199-1211, 2011.

[22] M. Farrell and S. Beaudoin, "Surface forces and protein adsorption on dextran- and polyethylene glycol-modified polydimethylsiloxane," Colloids and Surfaces B: Biointerfaces, vol. 81, no. 2, p. 468-475, 2010.

[23] J. Israelachvili, Intermolecular and Surface Forces, Academic Press, 2011.

[24] H. Wang and B.-m. Z. Newby, "Applicability of the extended Derjaguin-Landau-VerweyOverbeek theory on the adsorption of bovine albumin on solid surfaces," Biointerphases, vol. 9, p. 041006, 2014.

[25] C. J. Drummond and D. Y. C. Chan, "van der Waals Interaction, Surface Free Energies, and Contact Angles: Dispersive Polymers and Liquids," Langmuir, vol. 13, pp. 3890-3895, 1997.

[26] A. Koh, G. Gillies, J. Gore and B. R. Saunders, "Flocculation and Coalescence of Oil-inWater Poly(dimethylsiloxane) Emulsions," Journal of Colloid and Interface Science, vol. 
227, p. 390-397, 2000.

[27] D. Sameoto, H. Sharif and C. Menon, "Investigation of low-pressure adhesion performance of mushroom shaped biomimetic dry adhesives," Journal of Adhesion Science and Technology, vol. 26, no. 23, p. 2641-2652, 2012.

[28] R. H. J, Foundations of Colloid Science, Oxford, 2000.

[29] K. Mougin and H. Haidara, "Wetting of thin liquid films at nanoscale heterogeneous surfaces," EUROPHYSICS LETTERS, vol. 61, no. 5, p. 660-666, 2003.

[30] D. B. Hough and L. R. White, "The calculation of hamaker constants from liftshitz theory with applications to wetting phenomena," Advances in Colloid and Interface Science, vol. 14, no. 1, p. 3-41, 1980.

[31] S.-K. Chae, C.-H. Lee, S. H. Lee, T.-S. Kim and J. Y. Kang, "Oil droplet generation in PDMS microchannel using an amphiphilic continuous phase," Lab on a Chip, vol. 9, p. 1957-1961, 2009.

[32] K. Autumn, M. Sitti, Y. A. Liang, A. M. Peattie, W. R. Hansen, S. Spo, T. W. Kenny, R. Fearing, J. N. Israelachvili and R. J. Full, "Evidence for van der Waals adhesion in gecko setae," PNAS, vol. 99, no. 19, p. 12252-12256, 2002.

[33] Y.-L. Ong, A. Razatos, G. Georgiou and M. M. Sharma, "Adhesion Forces between E. coli Bacteria and Biomaterial Surfaces," Langmuir, vol. 15, pp. 2719-2725, 1999.

[34] C. T. H. Tran, A. Kondyurin, W. Chrzanowski, M. M. M. Bilek and D. R. McKenzie, "Influence of $\mathrm{pH}$ on yeast immobilization on polystyrene surfaces modified by energetic ion bombardment," Colloids and Surfaces B: Biointerfaces, vol. 104, p. 145-152, 2013.

[35] J. A. Brant and A. E. Childress, "Assessing short-range membrane-colloid interactions using surface energetics," Journal of Membrane Science, vol. 203, p. 257-273, 2002.

[36] C. J. van Oss, M. K. Chaudhury and R. J. Goods, "Interfacial Lifshitz-van der Waals and Polar Interactions in Macroscopic Systems," Chemical Reviews, vol. 88, pp. 927-941, 1988. 
[37] F. L. Leite, C. C. Bueno, A. L. Da Roz, E. C. Ziemath and O. N. Oliveira Jr, "Theoretical Models for Surface Forces and Adhesion and Their Measurement Using Atomic Force Microscopy," Int. J. Mol. Sci., vol. 13, no. Oliveira2012, pp. 12773-12856, 2012.

[38] S. H. Donaldson, A. Royne, K. Kristiansen, M. V. Rapp, S. Das, M. A. Gebbie, D. W. Lee, P. Stock, M. Valtiner and J. Israelachvili, "Developing a General Interaction Potential for Hydrophobic and Hydrophilic Interactions," Langmuir, vol. 31, no. 7, p. 2051-2064, 2015.

[39] A. J. Goldman, R. G. Cox and H. Brenner, "Slow viscous motion of a sphere parallel to a plane wall-II Couette flow," Chemical Engineering Science, vol. 22, no. 4, p. 653-660, 1967.

[40] Z. Adamczyk, B. Siwek, P. Warszynski and E. Musial, "Kinetics of Particle Deposition in the Radial Impinging-Jet Cell," Journal of Colloid and Interface Science, vol. 242, pp. 1424, 2001. 